\title{
The Role of the Frontal Pursuit Area in Learning in Smooth Pursuit Eye Movements
}

\author{
I-han Chou and Stephen G. Lisberger \\ Howard Hughes Medical Institute, Department of Physiology, and W. M. Keck Foundation Center for Integrative Neuroscience, University of California San \\ Francisco, San Francisco, California 94143-0444
}

The frontal pursuit area (FPA) in the cerebral cortex is part of the circuit for smooth pursuit eye movements. The present paper asks whether the FPA is upstream, downstream, or at the site of learning in pursuit eye movements. Learning was induced by having monkeys repeatedly pursue targets that moved at one speed for $150 \mathrm{msec}$ before changing speed. Single-cell recording showed no consistent correlate of pursuit learning in the responses of FPA neurons. Some neurons showed changes in firing in the same direction as the learning, others showed changes in the opposite direction, and many showed no changes at all. In contrast, the eye movements evoked by electrical stimulation of the FPA showed clear correlates of learning. Learning effects were observed when microstimulation was delivered during the initiation of pursuit and during fixation of a stationary target. In addition, learning caused changes in the degree to which stimulation of the FPA enhanced the eye velocity evoked by brief perturbations of a stationary target. The magnitude of the change in the stimulation-evoked eye movement in each tracking condition was proportional to the size of the eye movement evoked under that condition before learning. We conclude that learning occurs downstream from the FPA, possibly within the cerebellum, and that learning may be related to mechanisms that also control the gain of visual-motor responses on a rapid time scale.

Key words: motor cortex; gain control; visual-motor transformation; cerebellum; MT; MST

\section{Introduction}

Most motor actions are subject to learning. The circuits for motor control include many cortical and subcortical areas, but much attention has focused on the cerebellum as a site of motor learning. Physiological correlates of motor learning have been found in the cerebellum for the vestibulo-ocular reflex (VOR), classical conditioning of the eyelid response (for review, see Raymond et al., 1996), and smooth pursuit eye movements (Kahlon and Lisberger, 2000). The brainstem and cerebellar circuits that mediate VOR and the eyelid response are well studied, and much evidence points to the cerebellum as being the locus of learning for these reflexes. The circuits for smooth pursuit, however, are more extensive (Keller and Heinen, 1991), and the locus of learning has not been explored fully. Because the cerebral cortex is critical for accurate, high-gain pursuit (Dursteler and Wurtz, 1988; Keating, 1991; MacAvoy et al., 1991), it seemed plausible in principle that learning might occur through modification of signals in the cortex.

Previous examinations of patterns of learning generalization

Received Jan. 16, 2004; revised March 13, 2004; accepted March 15, 2004

This research was supported by the Howard Hughes Medical Institute and National Institute for Neurological Disorders and Stroke Grant NS34835. We thank Stefanie Tokiyama, Karen MacLeod, and Elizabeth Montgomery for assistance with the care of our monkeys and Scott Ruffner, Dirk Kleinhesselink, Ken McGary, and Lazslo Bocskai for their assistance with computer and equipment issues. We are grateful to members of the Lisberger laboratory for helpful comments and discussions.

Correspondence should be addressed to Stephen G. Lisberger, Department of Physiology, Box 0444, 513 Parnassus Avenue, Room 762-S, University of California San Francisco, San Francisco, CA 94143-0444. E-mail: sgl@phy.ucsf.edu.

DOI:10.1523/JNEUROSCI.0172-04.2004

Copyright $\odot 2004$ Society for Neuroscience $\quad$ 0270-6474/04/244124-10\$15.00/0 have suggested that learning occurs in a representation that is intermediate between purely sensory and purely motor, and that it is specific to the sensory-motor combination that is being altered (Kahlon and Lisberger, 1996; Chou and Lisberger, 2002). In addition, available evidence suggests that cortical and subcortical circuits generate pursuit through the interaction of two separate processes, either or both of which could be modified by learning. One process derives information about the direction and speed of image motion across the retina from the middle temporal (MT) and medial superior temporal (MST) areas of the extrastriate cortex and generates commands for changes in horizontal and vertical smooth eye velocity (Dursteler and Wurtz, 1988; Komatsu and Wurtz, 1988). The other process acts as a "gain control" that determines how strongly pursuit will respond to a given visual motion stimulus (Schwartz and Lisberger, 1994). Recent experiments from our laboratory have shown that output from the frontal pursuit area (FPA) in the arcuate sulcus can modulate the gain control: stimulation of the FPA increases the gain of smooth eye movements evoked by a given visual motion stimulus (Tanaka and Lisberger, 2001, 2002a). The gain control operates at an intermediate stage of visual-motor processing, and motor learning could be effected by alterations in the mechanisms that implement gain control.

Because the FPA seems to have direct access to the pursuit gain control, we tested the hypothesis that the gain control is the site of learning by exploring the relationship between FPA signals and motor learning in pursuit. We measured the effect of pursuit learning on the responses of neurons in the FPA and on the eye movements evoked by microstimulation in the FPA. Our results suggest that the site of learning is downstream from the FPA and 
support the hypothesis that alteration of the on-line gain control is responsible for pursuit learning. We suggest that gain control may be a general learning mechanism for recalibrating sensorimotor transformations and that it could be applicable to other motor systems.

\section{Materials and Methods}

General methods. We recorded from and stimulated the frontal pursuit area in two rhesus monkeys and tracked their eye movements with scleral search coils while they performed pursuit learning tasks. Details of how the animals were trained and prepared for physiological methods have been reported previously (Chou and Lisberger, 2002; Tanaka and Lisberger, 2002b). All surgical and behavioral procedures conformed to University of California San Francisco (UCSF) and federal guidelines on animal use and had been approved in advance by the Institutional Animal Care and Use Committee at UCSF.

Moving and stationary visual targets were presented using a mirrorgalvanometer projection system. Targets were created by imaging the light beam from a fiber-optic light source onto a pair of mirrors and projecting the beam onto the back of a large tangent screen placed $114 \mathrm{~cm}$ from the monkeys' eyes. The targets subtended $\sim 0.5^{\circ}$ in diameter, and the screen was $\sim 50 \times 40^{\circ}$. The movements of one eye were monitored using a scleral search coil system from CNC Engineering. All experiments were performed in dim ambient lighting.

Data acquisition and behavioral conditions were controlled by software running on a combination of a DEC Alpha UNIX workstation and a $500 \mathrm{MHz}$ Pentium-based PC running Windows NT and VenturCom RTX. The PC performed all real-time operations and controlled the visual displays, whereas the UNIX workstation provided a user interface for easy programming and modification of the experiment. Analog signals proportional to eye velocity were obtained by running the eye position voltages through an analog circuit that differentiated its inputs for frequencies up to $25 \mathrm{~Hz}$ and filtered higher frequencies with a roll-off of $20 \mathrm{~dB} /$ decade. Eye position, target position, and eye velocity signals were digitized at 1000 samples per second on each channel and stored, along with other codes related to the timing of trial events, for later analysis.

Behavioral paradigm for inducing pursuit learning. We used a behavioral protocol for inducing pursuit learning similar to that used by several previous studies (Kahlon and Lisberger, 2000). Pursuit trials began with the appearance of a stationary spot that the monkeys were required to fixate in the center of the screen. The spot then stepped $5^{\circ}$ eccentrically and began to move smoothly toward the fixation point. A complete experiment consisted of five blocks of trials. Each block consisted of a list of different types of target motions, some including stimulation. The list was repeated multiple times, shuffling the order of the trials before each repetition to ensure a random, interleaved design for stimulus presentation.

In the first, "baseline" block, we measured pursuit to a target moving at $20 \% \mathrm{sec}$ in the preferred direction for the site under study and in the opposite "control" direction (see Fig. 1 A, Probe and Control trials). Note that we use baseline to refer to the initial block of trials before learning and control to refer to trials that delivered target motion in the direction opposite to the learning direction.

In the second, "learning" block, we presented 100 consecutive learning trials, in which the moving target underwent an additional step in velocity $150 \mathrm{msec}$ after it began to move. In half the experiments, designed to cause learned increases in eye velocity, target motion started at $20 \% \mathrm{sec}$ and increased to $40 \% \mathrm{sec}$, as depicted in Figure $1 \mathrm{~B}$ (Learning trials). In the other experiments, designed to cause learned decreases in eye velocity, target motion started at $20 \% \mathrm{sec}$ and decreased to $5 \% \mathrm{sec}$. On any given day, we determined randomly whether the learning would deliver target motions designed to increase or decrease eye velocity at the initiation of pursuit.

In the third block of trials, we assayed learning by interspersing infrequent probe trials among the learning trials. Probe trials began like learning trials but did not include the additional step of target velocity 150 msec after the onset of motion. The probe trials revealed the extent of learning by delivering exactly the same target motions that we had used in the original baseline block of trials (see Fig. $1 \mathrm{~A}$, Probe and Control trials).
In the fourth, "reversal" block (see Fig. 1A, Reversal trials), we switched the learning trials. If the learning block had contained trials designed to cause learned increases in eye velocity, then the reversal block would contain trials designed to cause learned decreases in eye velocity, and vice versa.

The fifth and final block of trials again assayed learning, with probe trials intermingled among the reversal trials.

For experiments that involved microstimulation in the FPA, we included several additional trial types in the first, third, and fifth blocks. When it was included, stimulation began $100 \mathrm{msec}$ after the onset of target motion so that the stimulation-evoked smooth eye movement would occur during the first $100 \mathrm{msec}$ after the onset of pursuit. To assess the effects of learning on stimulation during fixation, we delivered the stimulation train while the monkeys fixated the central fixation spot. Finally, in 15 experiments, we included trials designed to test the interaction of learning, stimulation, and the on-line modulation of the gain of visual-motor transmission for pursuit (Tanaka and Lisberger, 2001; Churchland and Lisberger, 2002). These trials began by having the monkey fixate the central fixation spot for 1-2 sec. After a randomized amount of time, the target underwent a half-cycle of sinusoidal motion at $5 \mathrm{~Hz}$ with peak amplitude of $20 \% \mathrm{sec}$ in the preferred direction for the site. The perturbation lasted $100 \mathrm{msec}$ and caused a smooth displacement of the target by $0.63^{\circ}$. We timed the stimulation train so that the onset of the movement evoked by microstimulation coincided with the onset of the eye movement response to the sine wave of target motion. The target perturbations that we used were the same duration as those used previously (Tanaka and Lisberger, 2001), but they provided motion solely in the learning or control direction because they consisted of only a half cycle as opposed to a full cycle of sine wave motion.

Identification of the frontal pursuit area. The frontal pursuit area was identified physiologically using a combination of microstimulation and single neuron recording. Sites were considered to be within the FPA on the basis of the location of the recording site relative to the saccadic frontal eye fields, the presence of pursuit-related neurons, and the ability to evoke pursuit eye movements using microstimulation. Although histology is not available for either animal, the pursuit-related sites lay posterior to and deeper than sites from which saccades could be evoked with low currents $(<50 \mu \mathrm{A})$, conforming to several published descriptions of the location of the FPA in rhesus monkeys (MacAvoy et al., 1991; Tian and Lynch, 1996).

Microstimulation in the frontal pursuit area. Pursuit eye movements were evoked by applying current through tungsten electrodes (Frederick Haer, Bowdointon, ME) with impedances ranging from $800 \mathrm{~K} \Omega$ to 1.2 $\mathrm{M} \Omega$ measured at $1 \mathrm{kHz}$. Trains of biphasic pulses were applied, with total pulse duration of $0.2 \mathrm{msec}$, intensity of $70 \mu \mathrm{A}$, intra-train frequency of $333 \mathrm{~Hz}$, and train duration of $80 \mathrm{msec}$. We used several criteria to confirm that the movements were actually smooth pursuit and not lowvelocity saccades evoked by subthreshold current at saccadic sites or eye movements caused by facial movements. The criteria included enhancement of the size of stimulation-evoked movement when stimulation was applied during pursuit versus during fixation and increases in the duration of the stimulation-evoked movement with increasing train duration. We also increased current intensity, train frequency, and train duration to confirm that we were not weakly activating nearby saccade-related sites, and we monitored the animals with a video camera to determine whether stimulation caused pinna or facial movements.

Single-unit recordings. Action potentials were recorded from single units in the frontal pursuit area using electrodes similar to those used for stimulation, but with higher impedance (1.1-2 M $\Omega$ ). Neural activity was amplified, filtered with a bandpass of $200 \mathrm{~Hz}$ to $10 \mathrm{kHz}$, and passed to a dual-channel window discriminator to segregate and trigger the action potentials of single units. The times of spike occurrence were stored on the computer with a resolution of $10 \mu \mathrm{sec}$. The monkey performed pursuit and saccade trials as we advanced the electrode into the arcuate sulcus using a hydraulic microdrive (Narishige, Tokyo, Japan). When a neuron was encountered that showed modulation of firing rate in relation to pursuit, we characterized it on the basis of previously defined classifications of pursuit cells (Tanaka and Fukushima, 1998). We tested to see whether the neuron responded to pursuit only, to eye position, or 
to both pursuit and saccades, by having the monkey pursue in eight different directions, make saccades of various amplitudes, and maintain fixation at different positions. We conducted further analysis only for pursuitonly neurons $(n=24)$ and eye-position neurons $(n=1)$, because we wanted to analyze pursuit-related responses without the added complexity of saccadic modulation.

Once we had isolated a pursuit-related neuron, we recorded its response during pursuit of targets moving at $20 \% \mathrm{sec}$ in eight different directions. Preferred direction was determined by fitting a Gaussian function to graphs that plotted the mean firing rate as a function of the direction of pursuit. Pursuit velocity was averaged over the first $200 \mathrm{msec}$ of pursuit, but because most FPA pursuit-related neurons begin to discharge before the onset of pursuit, we averaged firing rate over $200 \mathrm{msec}$, beginning 50 msec before the onset of pursuit. Next, we recorded the responses of the neuron during pursuit in the preferred direction for targets of speeds ranging from 5 to $40 \% \mathrm{sec}$. To assess speed tuning, we plotted firing rate as a function of mean eye velocity. After characterizing the directional and speed tuning of the neuron, we performed the sequence of five sets of trials described above, using targets that moved in the cardinal direction closest to the preferred direction of the neuron for the learning and subsequent reversal trials. If the neuron remained isolated after a full set of learning, reversal, and probe blocks, we resampled directional and speed tuning to verify that we had recorded from the same neuron throughout.

Data analysis. For each trial that the monkey completed successfully, eye position and velocity were displayed on a computer screen, and saccades were marked using an automated saccade detection algorithm. The saccade delineations generated by the algorithm were checked individually and corrected by hand if necessary. All analyses of eye velocity and statistics were performed using Matlab (The Mathworks Inc.) and consisted primarily of computing average eye velocity traces for responses to identical target motions, aligned on the onset of target motion. Data from intervals that contained a saccade were omitted from the average. Thus, there could be a different number of samples in each bin of the average, and some bins might contain very few samples; we did not compute an average eye velocity unless a bin included data from at least eight trials. For recordings from neurons in the FPA, average spike rate during the initiation of pursuit was calculated by creating poststimulus spike histograms aligned on the onset of target motion and smoothing the histograms by convolving them with a Gaussian function $(\sigma=10$ msec).

\section{Results}

\section{Induction of pursuit learning}

Figure $1 A$ illustrates the effect of our learning protocol on pursuit eye movements. In the example shown, the learning stimulus consisted of a step increase in target velocity from 20 to $40 \% \mathrm{sec}$. When presented for 100 trials, the learning stimulus led to an increase in the eye velocity in the first $100 \mathrm{msec}$ of the evoked smooth eye velocity. The reversal stimulus consisted of a step decrease in target velocity from 20 to $5 \% \mathrm{sec}$ and, when presented for 100 trials, decreased the eye velocity in the first $100 \mathrm{msec}$ of the evoked smooth eye velocity. Learning was reflected in two ways. First, the eye velocity response to the learning stimulus grew as the experiment proceeded from the beginning (gray trace) to the end (black trace) of the learning block. Eye velocity began to
B
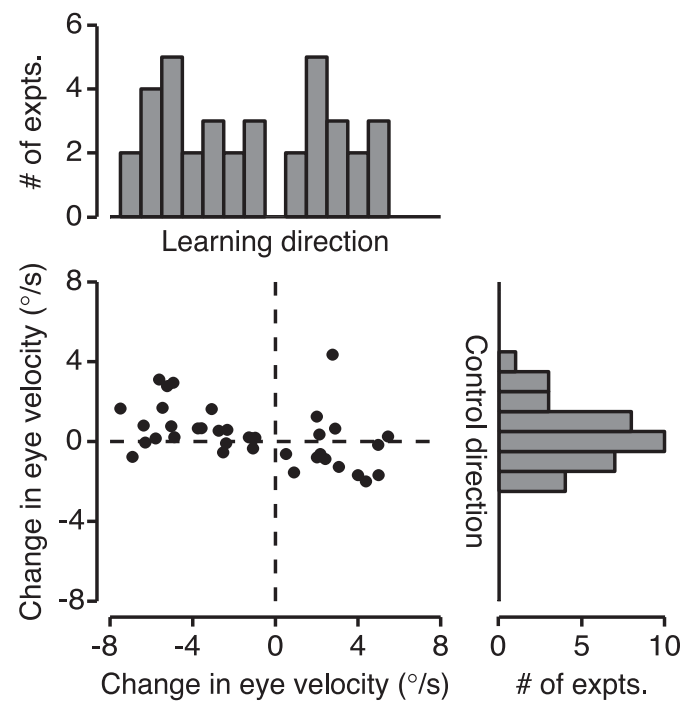

Figure 1. Expression of learning in smooth pursuit eye movements. $A$ (top to bottom), The superimposed traces show the time course of eye and target velocity for learning trials, reversal trials, probe trials, and control trials. The smooth traces with step 列 quantified by computing mean eye velocity in the first $200 \mathrm{msec}$ of pursuit and subtracting the value after the reversal block of trials from that after the learning block of trials. Data are plotted at positive or negative values on the $x$-axis depending on whether the learning block was designed to cause increases or decreases in eye velocity at the initiation of pursuit.

anticipate the second step of target velocity, so that it was larger than the baseline eye velocity almost from the beginning of the response to target motion at $20 \% \mathrm{sec}$. Eye velocity in the reversal block showed the opposite effect and declined from the start (bold trace) to the end (fine trace) of the block. Second, the response to probe target motion at $20 \% \mathrm{sec}$ was also modified by learning. Depending on whether the learning or reversal stimulus included an increase or decrease in target velocity, eye velocity was larger (bold black trace) or smaller (fine black trace) than the pre-learning baseline response (gray trace), and it tended to overshoot or undershoot target velocity, respectively. For all experiments, we analyzed the responses to the probe trials to assay learning.

In all 36 experiments, significant changes in eye velocity ( $t$ test; $p<0.05$ ) occurred in response to the first learning stimulus, regardless of whether the learned response was an increase or a decrease in eye velocity. In 32 of 36 experiments, the second learning stimulus successfully reversed the first learning effect, as illustrated in the example shown in Figure $1 A$. Although the second learning effect was perhaps less consistent than the first, for all subsequent analyses we compared measures obtained after the reversal block of trials with those obtained after the first learning block, instead of comparing the effects of individual learning conditions with responses in the baseline block. Our rationale was that we should look only for reversible changes, to ensure that any changes in physiology or behavior were specific to the pursuit learning. Also, experiments in which learning was unsuccessful or small provided us with another useful control for assessing whether the changes observed in other experiments were specific to learning.

Learning effects were specific to the direction of the learning 
A
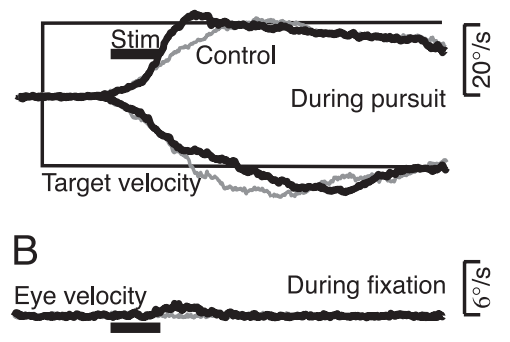

C

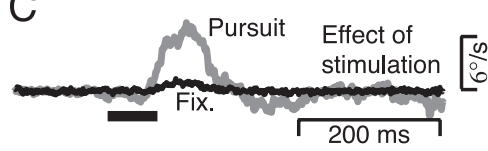

Figure 2. Methods for analyzing the effects of microstimulation in the FPA. A, Responses to stimulation during the initiation of pursuit. Rectangular traces show target velocity. Gray and black traces show the eye velocity at the initiation of pursuit without and with microstimulation in the FPA. Upward and downward deflections indicate target motion to the right or left. $B$, Responses to simulation during fixation of a stationary target. Black and gray traces show the eye velocity of fixation with and without stimulation of the FPA. C, The black and gray traces show the effect of microstimulation during pursuit and fixation, respectively. The black trace was obtained by subtracting the eye velocity without stimulation from that with stimulation at each time point of the averages for rightward pursuit in $A$. In $A-C$, the short horizontal black bar indicates the time when stimulation was applied. $D$, Effect of pursuit direction on the eye movements evoked by microstimulation in the FPA. Each pair of traces is plotted at a position indicating the direction of target and eye motion at the time of stimulation. Black and gray traces plot the horizontal and vertical eye velocity and were obtained by computing the difference eye velocity as eye velocity with stimulation minus that without microstimulation at each time point of the averages. Upward deflections indicate leftward and upward eye motion. Each vector in the plot at the center of $D$ shows the size and direction of the eye movement evoked by stimulation of the FPA during the initiation of pursuit for target motions indicated by the position of the vector. The central vector shows the eye movements evoked by stimulation during fixation.

stimulus. For example, for the experiment illustrated in Figure 1, the learning block did not induce changes in the eye velocity evoked by interleaved probe trials with target motion in the opposite, control direction (Fig. $1 A$, bottom traces). The graphs in Figure $1 B$ show the directional specificity of the behavioral learning across all experiments by plotting the change in mean pursuit velocity in the first $200 \mathrm{msec}$ of the response for target motion in the control direction (ordinate) as a function of that for target motion in the learning direction (abscissa). Figure $1 B$ shows a small negative regression slope of -0.16 that was significantly different from zero $(p<0.01)$, indicating that the pursuit in the control direction showed weak changes in the opposite direction from those for the learning direction, in agreement with the data of Kahlon and Lisberger (1996). In subsequent graphs, we use the change in eye velocity in the learning direction, in degrees per second, as our independent variable, and we call it the "magnitude of learning." Points are plotted with positive or negative signs on the abscissa, depending on whether the particular experiment began with an increase in learning followed by a decrease in reversal, or vice versa.

\section{Effect of learning on eye movements evoked by stimulation of} FPA during initiation of pursuit

We confirmed that sites lay within the FPA by applying microstimulation during the initiation of pursuit and during fixation using the strategy outlined in Figure $2 A-C$. As illustrated in Figure $2 A$, the initiation of pursuit during stimulation (continuous gray traces) was different from that without stimulation (dashed traces). For the site shown in Figure $2 A-C$, stimulation caused transiently higher eye velocities during the initiation of rightward pursuit (upward traces) and lower eye velocities during the initiation of leftward pursuit. For each condition, we measured the effect of stimulation by computing the "difference eye velocity," defined as the average eye velocity with stimulation minus that without stimulation. For the experiment shown in the left column of Figure 2, the difference eye velocity for stimulation during rightward pursuit was a substantial rightward transient deflection of eye velocity (Fig. 2C, continuous gray trace), followed by a small undershoot in the opposite direction. The source of this undershoot is unclear, but it is unlikely to be a visually driven response because its latency is too short. In contrast, stimulation during fixation of a stationary target usually caused only a small rightward deflection of eye velocity (Fig. $2 B$, continuous black trace).

Next, we characterized the directionality of the effect of microstimulation on eye velocity during the initiation of pursuit in eight directions. In Figure 2D, the pairs of individual traces show the effect of stimulation of the FPA on horizontal (black traces) and vertical (gray traces) eye velocity during pursuit of target motion in directions indicated by the placement of the traces. The vectors in the central inset summarize the effects of stimulation in polar coordinates: for this site, the largest effects occurred during target motion to the left and nearby directions. We then provided learning trials in the direction of the largest response, and all subsequent target motions in a given experiment were in either the learning direction or the opposite direction along the same axis. We identified sites using the stimulation parameters described in Materials and Methods, but we were concerned that learning effects might be masked by high current intensity (Edelman and Goldberg, 2002). To address this issue, whenever possible we decreased the current intensity to a point at which smooth eye movements were still evoked, but with lower amplitude, before performing our learning paradigms. Across all experiments, current intensity ranged from 30 to $70 \mu \mathrm{A}$.

The three columns in Figure 3 show the results of three example experiments in which learning affected the eye movements evoked by microstimulation in the FPA. In each experiment, the stimulation-evoked eye movements varied in parallel with learning. In the left column, the sequence of learning was designed first to increase and then decrease eye velocity during the initiation of pursuit. Analysis of pursuit behavior without microstimulation (Fig. $3 A$ ) revealed an increase in eye velocity for probe trials after the first learning block (bold black trace) relative to the prelearning probe trials (gray trace), and a return to baseline responses after the reversal block (dashed trace). The difference eye velocity evoked by microstimulation during probe trials showed the same pattern (Fig. 3D): the peak-to-peak response was larger after the learning block (bold black trace) than in the baseline block (gray trace) or after the reversal block (dashed trace). The same general pattern is visible in the other two experiments, in which the learning block was designed to cause an increase (Fig. $3 B, E$ ) or a decrease (Fig. 3C,F) in eye velocity. For consistency, the eye velocities after the learning and reversal blocks are always shown as black or dashed traces, respectively, to reveal the order in which the experiment was run. Thus, the learning and reversal blocks caused the eye velocity in the initiation of pursuit to in- 
crease and then decrease in Figure 3, $A, B, D$, and $E$, and to decrease and then increase in Figure $3, C$ and $F$. The effects of learning on the difference eye velocity evoked by microstimulation followed the effects on the initiation of pursuit and therefore were reversed in Figure 3, $C$ and $F$. For all three experiments illustrated in Figure 3, learning had small effects on the eye movements evoked by microstimulation during fixation (Fig. $3 G-I$ ), which themselves were small even in the baseline blocks.

Figure 4 summarizes the effect of pursuit learning on the eye movements evoked by microstimulation in the FPA for all 36 experiments. For the analysis of responses to microstimulation during pursuit (Fig. $4 A, B$ ), we analyzed the responses to target motion during pursuit in the preferred direction for the stimulation site (filled circles), which was used as the learning direction, and for the opposite, control direction (open triangles). We quantified the amount of behavioral learning in each experiment by computing the mean eye velocity during the first 200 msec of pursuit during probe trials that followed the first learning block minus that during probe trials that followed the reversal block. By assembling the data from all our experiments in graphs like those in Figure 4, we could assess the relationship between the size of the behavioral learning and the size of the effect of learning on the eye movements evoked by stimulation in the FPA. When we measured the peak eye velocity induced by microstimulation during the initiation of pursuit, the regression slope was 0.13 for stimulation during pursuit in the preferred direction (Fig. $4 A$, filled circles) ( $p=$ 0.12 ). When we measured the peak-topeak eye velocity from the same data, the regression slope was 0.60 for stimulation during pursuit in the preferred direction of the site (Fig. $4 B$, filled circles) $(p<0.01)$. In contrast, the relationship between peak eye velocity during pursuit in the control direction and learning had low slopes that were not significant for either measure [Fig. $4 A$, open symbols (slope $0.01, p=0.85$ ); Fig. $4 B$, open symbols (slope $=0.11, p=$ $0.08)]$. There was a weak but statistically significant relationship between the size of the eye movement evoked by stimulation in the FPA during fixation and the size of the behavioral learning, with a regression slope of 0.06 (Fig. $4 C)(p<0.005)$.

\section{Interaction of pursuit learning, on-line gain control for} pursuit, and microstimulation in the FPA

We have shown previously that the output of the FPA has access to the setting of a gain control that operates in the conversion of visual motion inputs to motor outputs, because microstimulation of FPAincreases the magnitude of pursuit responses to visual motion (Tanaka andLisberger, 2001). To address whetherlearning interacted with the gain control, we examined the pursuit evoked by brief sine wave motion of an otherwise stationary tar- get (Fig. 5, fine traces) in the preferred and opposite directions for a site. In the absence of stimulation in the FPA, responses to the perturbation (Fig. $5 B$, gray traces) had gains of $0.2-0.5$, consistent with previous observations (Schwartz and Lisberger, 1994). If the brief target motion was paired with microstimulation in the FPA, then the response to the perturbations was increased (Fig. $5 B$, black traces), consistent with Tanaka and Lisberger (2001). Importantly, the enhancement was present for perturbations in both directions, showing that the microstimulation is enhancing the response to the visual stimulus and not vice versa.

To quantify the amount of enhancement of the response to the brief perturbation of target motion in each state of pursuit learning, we performed a two-step analysis. (1) For each millisecond in the averages of the eye velocity responses, we removed the eye velocity evoked directly by microstimulation alone by subtracting the eye velocity response for microstimulation during fixation (Fig. $5 \mathrm{C}$ ) from the response to the combination of the perturbation and stimulation. (2) We subtracted the eye velocity response to the perturbation in the absence of stimulation, leaving only the enhanced component of the response. 
A
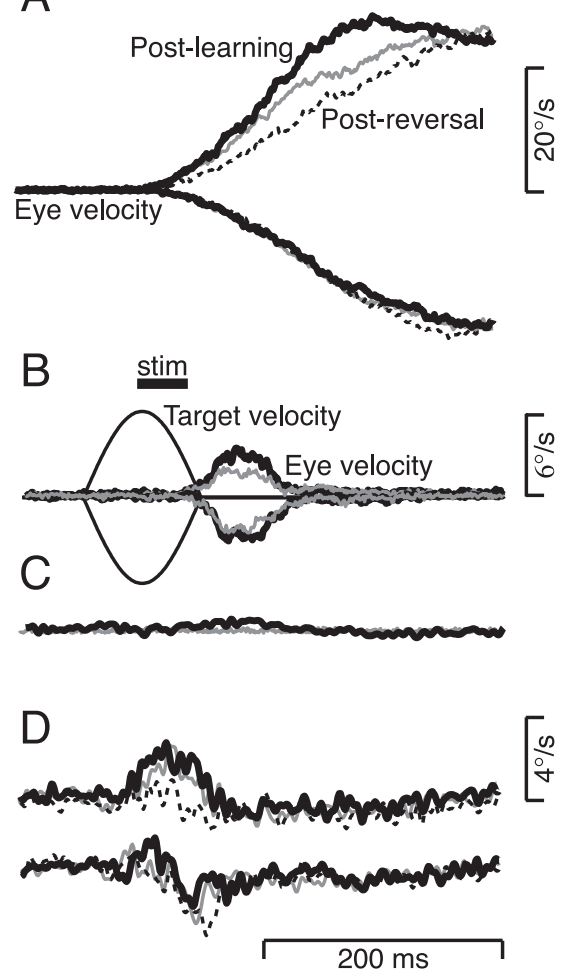
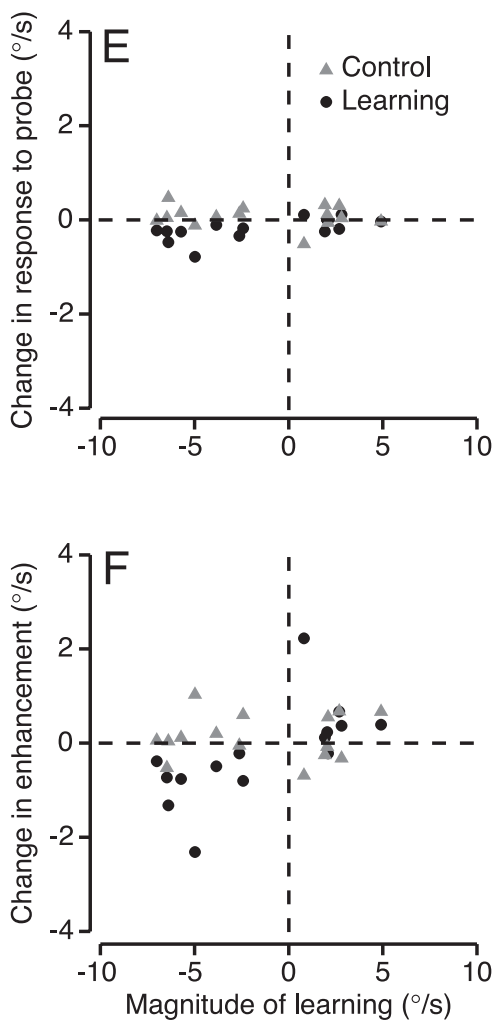

Figure 5. Effect of learning on the multiplicative interaction of microstimulation in the FPA and visual inputs caused by brief target motions. $A$, Average eye velocity traces showing the expression of learning in the initiation of pursuit. Gray, bold, and dashed traces show responses in baseline probe trials (pre-learning), post-learning probe trials, and post-reversal probe trials. $B_{i}$ The smooth half-sine waves show the target velocity used to provide perturbations of target motion, and the rougher traces show averages of eye velocity evoked by the perturbations. Bold and thin traces show responses in the presence and absence of stimulation in the FPA. C, Bold and fine traces show average eye velocity evoked by microstimulation during fixation and the baseline during fixation. $D$, Enhanced component of eye velocity, computed by subtracting the response to the perturbation during fixation from that during stimulation at every millisecond in the averages. As in $A$, gray, bold, and dashed traces show responses in baseline probe trials (pre-learning), post-learning probe trials, and post-reversal probe trials. E, F, Two points are plotted for each experiment in each graph. Each point shows the effect of learning on the response to the perturbation as a function of the change in eye velocity at the initiation of pursuit. $E$, Responses to perturbations in the absence of stimulation. $F$, Responses to perturbations combined with stimulation of the FPA. Black circles and gray triangles show responses to perturbations in the learning and control directions, respectively. All effects of learning were calculated as the response in the post-learning probe block minus the response in the post-reversal probe block.

We then compared the isolated enhanced component of the responses to the perturbation in the baseline, post-learning probe block, and post-reversal probe blocks (Fig. 5D). For this example, learning caused a consistent, if small, effect on the size of the microstimulation-induced enhancement of the response to the brief target perturbations in either direction. The pursuit learning itself is documented in Figure 5A. The sequence of learning and reversal blocks caused first an increase (bold trace) and then a decrease (dashed trace) in the eye velocity evoked by target motion at $20 \% \mathrm{sec}$. The results of 15 experiments are summarized in Figure 5, $E$ and $F$. Each graph plots the effect of learning on the response to the target perturbation as a function of the magnitude of learning in the initiation of pursuit for target motion in the learning direction. Learning had a small but significant effect on the peak eye velocity of the response to the perturbation in the absence of stimulation of the FPA (Fig. 5E) for perturbations in the learning direction (black circles; regression slope, $0.03 ; p<$ 0.05 ) but not the control direction (gray triangles; regression slope, $-0.01 ; p=0.6$ ). In contrast, learning caused a larger effect on the size of the enhanced response to delivery of the target perturbation during stimulation in the FPA (Fig. $5 F$ ), but only when the direction of the perturbation coincided with the learning direction. For perturbations that provided target motion in the learning direction, the regression slope was 0.16 (black circles; $p<0.01$ ); for perturbations in the opposite, control direction, the regression slope was 0.008 (gray triangles; $p=0.8$ ).

\section{Relationship between expression of learning and size of baseline response} Figures 4 and 5 show that different experimental conditions yielded very different slopes of the relationship between the size of the expression of learning in a given behavioral condition and the magnitude of behavioral learning: slopes were 0.03 , $0.06,0.13,0.16$, and 0.60 when we measured the effect of learning on the eye velocities evoked, respectively, by (1) perturbations of the target without stimulation, (2) stimulation during fixation, (3) stimulation during the initiation of pursuit (peak only), (4) stimulation combined with perturbations, and (5) stimulation during the initiation of pursuit (peak to peak).

We also found a strong and consistent relationship between the amount of change in response as a result of learning and the size of the baseline eye movement response. In Figure 6, the filled circles represent responses averaged across the 15 stimulation sites in which we were able to study the effects of learning on the responses to stimulation of the FPA in five different behavioral conditions that used three target-motion conditions: fixation, initiation of pursuit for step-ramp target motion, and sine wave perturbations of target motion. Each circle summarizes the averages for a different behavioral condition and plots the average magnitude of the effect of learning on the eye movements as a function of the size of the baseline responses under those conditions. From left to right, the behavioral conditions for the five points were as follows: perturbations delivered during fixation without stimulation of the FPA; stimulation during fixation; perturbations delivered during fixation with stimulation of the FPA; peak responses for stimulation delivered during the initiation of pursuit; and peak-to-peak responses delivered during the initiation of pursuit. On average, larger baseline responses were associated with larger expressions of learning. The same relationship is apparent in the 75 symbols not connected by lines in Figure 6 that plot the results from each experiment separately (five behavioral conditions times 15 stimulation sites). For the entire set of points, regression analysis yielded a highly significant slope of $0.43(p<0.01 ; r=0.63)$. Within each of the groups for the five different behavioral conditions (Fig. 6, different symbols), neither the slope from the regression analysis nor the correlation coefficients reached statistical significance. Inspection of the scatter plot in Figure 6 shows that this failure simply reflects the narrow range of values of baseline response magnitude within each behavioral condition. 


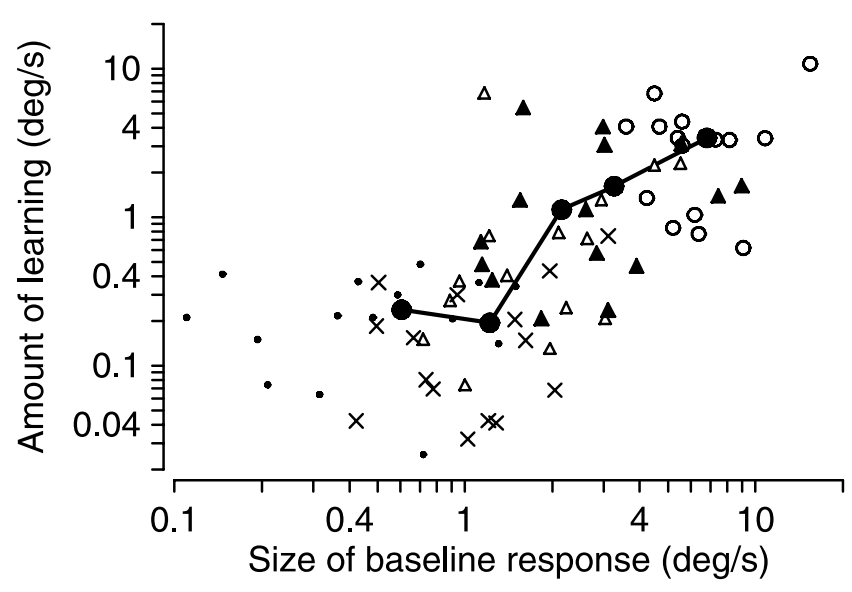

Figure 6. Relationship between the magnitude of the effect of learning on eye movements evoked by microstimulation in the FPA and the size of the baseline response. Individual symbols not connected by lines show responses for each of 5 stimulus conditions at 15 different stimulation sites. Large circles connected by lines show means across the 15 stimulation sites for each of the 5 stimulus conditions. From left to right, the five large filled circles and their associated individual symbols provide data for perturbations of target motion delivered during fixation without stimulation (small dots), stimulation of the FPA during fixation ( $X$ symbols), perturbations delivered during fixation with stimulation of the FPA (open triangles), peak responses for stimulation delivered during the initiation of pursuit (filled triangles), and peak-to-peak responses delivered during the initiation of pursuit (open circles). The graph is plotted in log-log coordinates to facilitate visualization of the data.

\section{Effect of learning on neural responses in FPA}

Most FPA neurons have firing rates that increase monotonically as a function of increasing pursuit speed in their preferred direction (Gottlieb et al., 1994; Tanaka and Lisberger, 2001). For such neurons, if learning occurs within FPA, we would expect to record larger responses during test trials after learning-induced increases in pursuit eye velocity and smaller responses after learning-induced decreases. We recorded from 25 pursuitrelated neurons in the FPA of two monkeys while they performed the same sequence of learning and reversal blocks described above. Learning was induced for target motion in the preferred direction of the neuron under study, and test trials provided target motion at $20 \% \mathrm{sec}$ in the learning direction. Figure $7 \mathrm{~A}$ shows the response of one neuron during test trials in the baseline block before learning (top row of rasters and gray traces) and in the probe blocks after learning-induced increases in eye velocity at the initiation of pursuit (middle row of rasters and bold black traces) and reversal-induced decreases in eye velocity (third row of rasters and dashed traces).

For the example experiments illustrated in Figure $7 A$, the eye velocity traces show that the learning paradigms caused the expected changes in motor output, with increases in the eye velocity during the initiation of pursuit after the learning block (black traces) and decreases after the reversal block (dashed traces); however, we did not observe consistent changes in neuronal firing rate. The average firing rates showed an increase in responsiveness between the baseline block (gray trace) and the postlearning probe block (black traces), but the neural response did not decline when the pursuit response declined after the reversal block (dashed trace).

Across all neurons, we did not find a consistent relationship between changes in the size of neural responses and learned changes in eye velocity. To assess the size of the neural responses, we measured the mean firing rate during the $200 \mathrm{msec}$ interval that began $50 \mathrm{msec}$ before the onset of pursuit in the postlearning and post-reversal test trials, and we estimated the change in the size of the neural response as the difference between the means. To assess the amount of behavioral learning, we measured the mean eye velocities in the first $200 \mathrm{msec}$ of pursuit and computed the eye velocity in the post-reversal probe trials minus that in the post-learning trials. Plotting the change in the neural response as a function of the amount of behavioral learning for all 25 FPA neurons failed to show a consistent relationship (Fig. 7C) (regression slope, $0.39 ; p=0.59$ ). This result was independent of the analysis interval used for firing rate, which was varied from as short as the initial transient response to the full $500 \mathrm{msec}$ of response.

The analysis on the right side of Figure 7 is modeled after an analysis done for data obtained from Purkinje cells in the floccular complex after pursuit learning (Kahlon and Lisberger, 2000). We have used the same data presented on the left side of the figure, but we now ask whether the changes in neural responses are larger, smaller, or the same as what would be predicted by considering how the learned change in eye velocity would move the response along the baseline relationship between firing rate and eye velocity. Might this explain the apparently random relationship between changes in the firing of FPA neurons and the amount of behavioral learning in Figure $7 C$ ? For each neuron, we constructed a speed tuning curve from data obtained in the prelearning baseline block of trials by measuring firing rate during pursuit of target motion at six different speeds ranging from 5 to $40 \% \mathrm{sec}$. We plotted the mean firing rate as a function of the mean eye velocity during the first $200 \mathrm{msec}$ of pursuit (Fig. 7B, open gray circles) and fitted the data with either a sigmoid or linear function using nonlinear least squares regression; for each neuron, we chose the function that provided the best fit to the data with the lowest residuals. In our population of 25 neurons, most showed monotonic increases in firing rate as a function of eye velocity (Fig. $7 B$ ), but a few showed decreases or little relationship between firing rate and eye velocity during the initiation of pursuit.

We then plotted three points on the same graph that represented the response of the neuron as a function of the mean eye velocity in the initiation of pursuit for the probe trials in the baseline, post-learning, and post-reversal blocks (Fig. 7B, black squares). The neuron illustrated in this example was unusual in that the effect of learning on its firing exceeded the predictions from the baseline speed tuning curve. When the initial eye velocity of pursuit was decreased by learning (left-most black square), the response of the neuron decreased more than predicted from the baseline speed tuning curve. When the initial eye velocity of pursuit was increased by learning (right-most black square), the response of the neuron increased exactly as predicted from the baseline speed tuning curve.

To summarize the relationship between the actual and predicted effects of learning on the responses of our full sample of FPA neurons, we created the scatter plot in Figure 7D: each point plots data from one of our 25 neurons. For each neuron, we computed (1) the actual change in response as the firing rate after the reversal block of trials minus that after the learning block (Fig. $7 C$, " $\Delta$ actual") and (2) the predicted change as the difference between the firing rates predicted from the baseline speed tuning function for the measured effects of learning on the initiation of pursuit (Fig. $7 C$, " $\Delta$ predicted"). Plotting the actual change as a function of the predicted change failed to reveal a consistent trend across the population. Some neurons plotted along the vertical line, indicating large effects of learning on neural responses despite a prediction of no effect; other neurons plotted along the horizontal line, indicating no effect of learning on neu- 


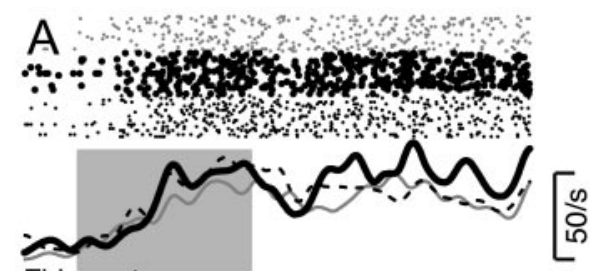

Firing rate
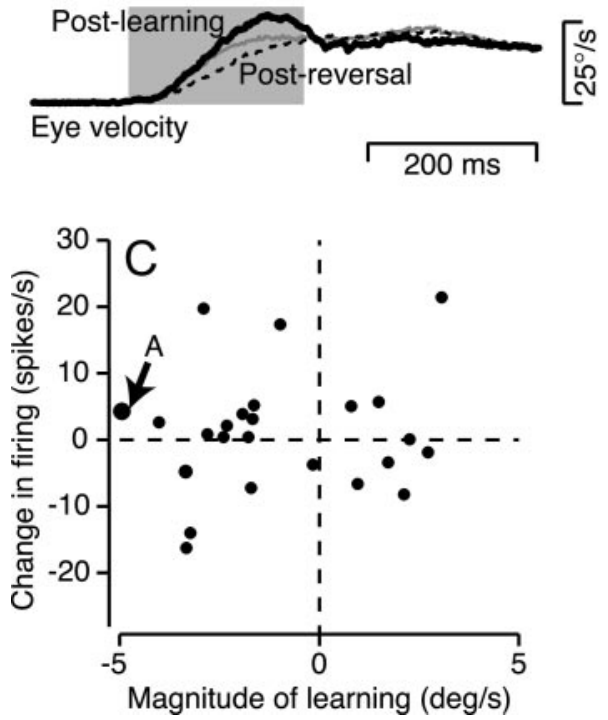
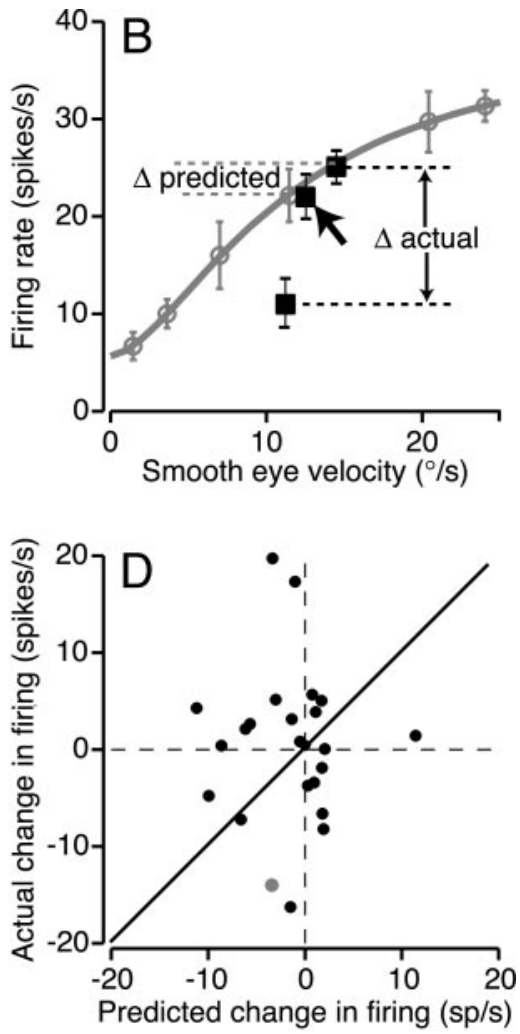

Figure 7. Absence of effect of learning on the responses of neurons in the FPA during the initiation of pursuit. $A$, Example responses of one neuron. From top to bottom, three rasters show pre-learning, post-learning, and post-reversal responses. Middle and bottom sets of traces show average firing rate and eye velocity during probe trials. Gray, bold, and dashed traces show responses in pre-learning, post-learning, and post-reversal probe trials. $B$, Method for assessing effect of learning on responses of FPA neurons in the context of the baseline relationship between firing rate and eye velocity in the initiation of pursuit. Gray symbols connected by a curve show the responses to targets moving at different speeds before learning. The three black squares show responses in probe trials before learning and after the learning and reversal blocks. Arrow points to the pre-learning response. $\Delta$ actual and $\Delta$ predicted indicate the changes in firing rate actually caused by learning and that predicted from the gray, speed tuning curve. C, Each point shows the response of a single FPA neuron and plots the effect of learning on firing rate as a function of the change in eye velocity at the initiation of pursuit. Arrow labeled " $\mathrm{A}$ " points to the analysis for the neuron illustrated in $A$. D, Each point plots the response of a single FPA neuron and shows the actual change in firing rate caused by learning as a function of that predicted by the speed tuning curve, using the analysis outlined in $B$. The horizontal and vertical dashed lines indicate predicted and actual changes of zero, and the line of slope one indicates where points should plot if the predicted and actual changes in response are identical. All effects of learning were calculated as the response in the post-learning probe block minus the response in the post-reversal probe block.

ral responses despite a prediction of a large effect. The neuron used to create Figure $7 C$, shown as a gray circle, is one of the exceptions in which the predicted and actual changes in firing were even in the same direction. We conclude that learned changes in the gain of pursuit are not represented in any consistent way in the firing rate of the full population of pursuit-related neurons in the FPA.

\section{Discussion}

Our analysis of the relationship between the FPA and learning in smooth pursuit eye movements has revealed a number of new findings. (1) Unlike the positive result found in the floccular complex of the cerebellum (Kahlon and Lisberger, 2000), learned changes in pursuit were not reflected in the responses of the population of FPA neurons. (2) Learning affects the eye velocity induced by microstimulation of the FPA under various targetmotion conditions. (3) In general, the effect of learning on the eye movements evoked by stimulation of the FPA is direction selective and appears during target motion in the learning, but not the control, direction. (4) For the learning direction, the size of the changes in stimulation-evoked eye movements is closely related to the size of the baseline eye movement. If the eye movement is small, it shows small changes; if the eye movement is large, it shows large changes.

\section{Magnitude of learning effects}

We designed our experiments and our data analysis on the premise that proper controls and the noise immunity of our measurements of the effects of learning mattered more than the size of the effect. The effects of learning on stimulationevoked eye movements were small but robust and consistently paralleled learned changes in pursuit velocity. There are three ways by which we might have increased the size of the learning effects, each with some cost. First, we could have induced the larger magnitudes of pursuit learning seen in previous studies (Chou and Lisberger, 2002) by delivering a larger number of consecutive learning trials. Instead, however, we chose to look for reversible effects by comparing responses after learning in one direction with responses after reversal of learning in the other direction. By comparing responses after learning in one direction with responses after reversal of learning in the other direction, we can be more confident that the changes that we observed were really related to learning and not simply a consequence of unidirectional drift in baseline responses. Second, because the magnitude of the effect of learning was related to the size of the baseline movement evoked by stimulation in the FPA, we could also have increased our learning effects, for example, by increasing current intensity or stimulus duration. We chose, however, conservative stimulation parameters to increase the probability that the efficacy of stimulation would not degrade, because of either tissue or electrode damage, over the course of an experimental session. Third, we could have made the responses appear larger by measuring the peak eye acceleration or peak firing rate in the initiation of pursuit instead of averaging eye velocity and firing rate over 200 msec intervals. By averaging across time, we did decrease the magnitude of the effects of learning, but we also reduced measurement noise.

\section{Locus of pursuit learning within the conceptual signal flow for pursuit}

Current ideas about the neural mechanisms of pursuit emphasize the existence of two separate conceptual pathways that perform quite different computations. One pathway originates in MT and provides visual signals that are used to drive changes in eye velocity, measured as eye accelerations. The other pathway modulates the gain of transmission of visual signals to the motor system. There is now extensive evidence in favor of the existence of a gain control in pursuit, showing a number of instances in which initial 


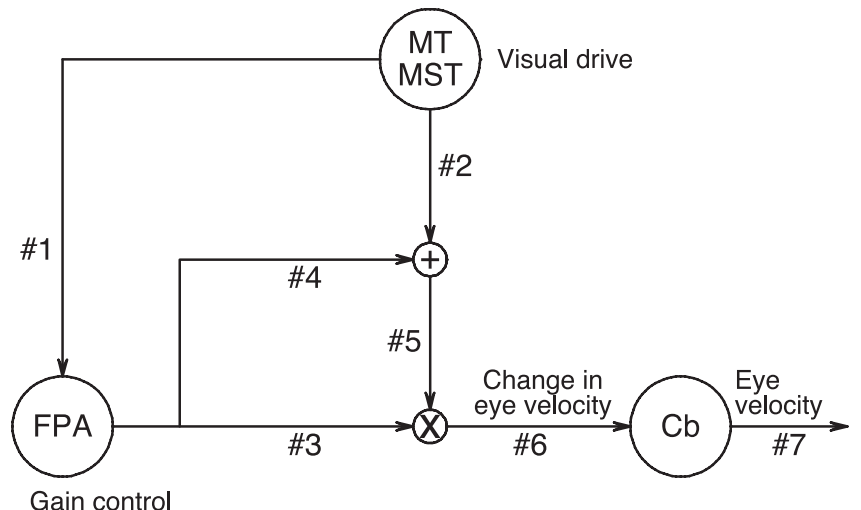

Figure 8. Schematic diagram showing the conceptual organization of the pursuit system. Signals flow along the arrows, which are numbered to allow easy reference in the text. Circles labeled MT/MST, FPA, and Cb are intended to represent extrastriate visual areas MT and MST, the frontal pursuit area, and the cerebellum. The circles with a plus sign or an $X$ in them perform addition or multiplication.

conditions alter the size of the motor response to a given visual stimulus (Schwartz and Lisberger, 1994; Tanaka and Lisberger, 2000; Missal and Heinen, 2001; Churchland and Lisberger, 2002). The output from the structure that we have studied here, the FPA, appears both to provide commands that drive smooth eye velocity and to play an important role in controlling the setting of the gain control (Tanaka and Lisberger 2001, 2002a).

We have instantiated this simple conceptual model in Figure 8 , with each signal pathway of the model numbered to facilitate discussion of its possible role in learning. Visual drive arises from MT/MST (arrow 2) and gain control arises from the frontal pursuit area (arrow 3). Gain control is represented as a multiplication junction, shown by the circle with an " $X$ " in it. Because stimulation of the frontal pursuit area evokes smooth eye movements during fixation, when there is no visual drive for pursuit, the output of the FPA must both drive eye movement and modulate visual-motor transmission. The drive arises from sidebranch 4, which adds to the visual drive from MT/MST, although the separation of arrows 3 and 4 in the model is not meant to imply necessarily separate anatomical pathways in the brain. The output from the multiplication junction (arrow 6) provides a command for a change in eye velocity, which is integrated by the cerebellum $(\mathrm{Cb})$ to drive the actual eye velocity (arrow 7). Finally, corticocortical pathways provide input from MT/MST to the FPA (arrow 1). Although the model in Figure 8 is different in detail, it has the same basic flow of signals proposed in Tanaka and Lisberger (2002a, their Fig. 17).

Our data exclude the possibility that learning occurs in the visual inputs to the pursuit system (sites 1 and 2), because learning at either of those sites should not cause the changes that we have demonstrated in the eye movements evoked by stimulation of the FPA. In addition, learning at site 1 would be expected to cause neurons in the FPA to change their responses in association with pursuit learning, which they do not. Site 7 is also excluded because it would cause learning to be expressed in eye velocity commands (site 7), causing changes that we did not observe in the steady-state eye velocity well after the initiation of pursuit.

One of the key observations from our FPA stimulation data sheds some light on the intermediate representation of the impending eye movement where learning seems to occur. Learninginduced changes in the size of the eye movements evoked by stimulation of the FPA are in direct proportion to the size of the baseline responses. For example, the eye movement evoked by stimulation during fixation was small and underwent small changes; peak-to-peak eye velocity evoked by stimulation during pursuit initiation was large and underwent the largest changes. A change that scales with the size of the baseline response is a change in gain and would be consistent with the hypothesis that learning is implemented by changing the multiplication that we use to represent the gain control. In Figure 8, the sites denoted as 3-6 all come into immediate contact with the gain control, and all are potential sites of learning.

Locus of learning within the anatomical pathways for pursuit The present study supports the previous hypothesis that pursuit learning occurs in a representation that probably is neither in the sensory representation of cortical area MT nor in the motor representation of the final brainstem oculomotor pathways. Correlates of learning have been observed in the responses of Purkinje cells in the floccular complex of the cerebellum (Kahlon and Lisberger, 2000) but could have been inherited from a site of learning in the cerebral cortex, as postulated for other forms of motor learning, such as adaptation of arm movements to changes in the forces within a dynamical work space (Li et al., 2001). Our data argue against a site of learning in the cerebral cortex for pursuit. We did not observe learning in the responses of neurons in the FPA, a "motor area" within the pursuit circuit, yet we did find learning-induced changes in the eye movements evoked by microstimulation in the FPA. Thus we conclude that learning probably occurs downstream from the FPA and that gain control and learning both occur in subcortical circuits that are interposed between the cortical outputs from areas MT, MST, and FPA and the cerebellum. We note that this conclusion could be wrong if the outputs from the FPA to the subcortical pursuit system emanate only from the small subgroup of FPA neurons that showed the "correct" changes in firing rate in association with pursuit learning.

Our data are consistent with, but do not prove, the hypothesis that pursuit learning occurs in the cerebellum, as is the case for other eye movements such as saccades (Optican and Robinson, 1980; Desmurget et al., 2000; Scudder, 2002) and the vestibuloocular reflex (Raymond et al., 1996). Learning could occur in brain areas that lie intermediate to the cortex and cerebellum. First, a major output from the FPA projects to a region of the caudate nucleus that shows high levels of activity in positron emission tomography scans taken during pursuit (O'Driscoll et al., 2000; Cui et al., 2003). Second, MT, MST, and the FPA all project to the dorsolateral pontine nuclei (Suzuki et al., 1990; Glickstein et al., 1994), which carry both visual and pursuitrelated signals (Suzuki and Keller, 1984; Suzuki et al., 1990) that in turn project to pursuit-related regions of the cerebellum. It is not known how pursuit signals are transformed in this relay, or whether gain control and learning could occur there. Finally, our thinking does not incorporate roles for recurrent feedback from both the cerebellum and the basal ganglia to the cerebral cortex, both of which are present in anatomical data (Tian and Lynch, 1997) and in the physiological responses of FPA neurons (Tanaka and Lisberger, 2002c). Future work on the neural control of pursuit eye movements will need to consider all of these features of the pursuit circuits and their possible roles in learning and gain control.

\section{References}

Chou IH, Lisberger SG (2002) Spatial generalization of learning in smooth pursuit eye movements: implications for the coordinate frame and sites of learning. J Neurosci 22:4728-4739. 
Churchland AK, Lisberger SG (2002) Gain control in human smoothpursuit eye movements. J Neurophysiol 87:2936-2945.

Cui DM, Yan YJ, Lynch JC (2003) Pursuit subregion of the frontal eye field projects to the caudate nucleus in monkeys. J Neurophysiol 89:2678-2684.

Desmurget M, Pelisson D, Grethe JS, Alexander GE, Urquizar C, Prablanc C, Grafton ST (2000) Functional adaptation of reactive saccades in humans: a PET study. Exp Brain Res 132:243-259.

Dursteler MR, Wurtz RH (1988) Pursuit and optokinetic deficits following chemical lesions of cortical areas MT and MST. J Neurophysiol 60:940-965.

Edelman JA, Goldberg ME (2002) Effect of short-term saccadic adaptation on saccades evoked by electrical stimulation in the primate superior colliculus. J Neurophysiol 87:1915-1923.

Glickstein M, Gerrits N, Kralj-Hans I, Mercier B, Stein J, Voogd J (1994) Visual pontocerebellar projections in the macaque. J Comp Neurol 349:51-72.

Gottlieb JP, MacAvoy MG, Bruce CJ (1994) Neural responses related to smooth-pursuit eye movements and their correspondence with electrically elicited smooth eye movements in the primate frontal eye field. J Neurophysiol 72:1634-1653.

Kahlon M, Lisberger SG (1996) Coordinate system for learning in the smooth pursuit eye movements of monkeys. J Neurosci 16:7270-7283.

Kahlon M, Lisberger SG (2000) Changes in the responses of Purkinje cells in the floccular complex of monkeys after motor learning in smooth pursuit eye movements. J Neurophysiol 84:2945-2960.

Keating EG (1991) Frontal eye field lesions impair predictive and visuallyguided pursuit eye movements. Exp Brain Res 86:311-323.

Keller EL, Heinen SJ (1991) Generation of smooth-pursuit eye movements: neuronal mechanisms and pathways. Neurosci Res 11:79-107.

Komatsu H, Wurtz RH (1988) Relation of cortical areas MT and MST to pursuit eye movements. I. Localization and visual properties of neurons. J Neurophysiol 60:580-603.

Li CS, Padoa-Schioppa C, Bizzi E (2001) Neuronal correlates of motor performance and motor learning in the primary motor cortex of monkeys adapting to an external force field. Neuron 30:593-607.

MacAvoy MG, Gottlieb JP, Bruce CJ (1991) Smooth-pursuit eye movement representation in the primate frontal eye field. Cereb Cortex 1:95-102.

Missal M, Heinen SJ (2001) Facilitation of smooth pursuit initiation by electrical stimulation in the supplementary eye fields. J Neurophysiol 86:2413-2425.

O’Driscoll GA, Wolff AL, Benkelfat C, Florencio PS, Lal S, Evans AC (2000)
Functional neuroanatomy of smooth pursuit and predictive saccades. NeuroReport 11:1335-1340.

Optican LM, Robinson DA (1980) Cerebellar-dependent adaptive control of primate saccadic system. J Neurophysiol 44:1058-1076.

Raymond JL, Lisberger SG, Mauk MD (1996) The cerebellum: a neuronal learning machine? Science 272:1126-1131.

Schwartz JD, Lisberger SG (1994) Initial tracking conditions modulate the gain of visuo-motor transmission for smooth pursuit eye movements in monkeys. Vis Neurosci 11:411-424.

Scudder CA (2002) Role of the fastigial nucleus in controlling horizontal saccades during adaptation. Ann NY Acad Sci 978:63-78.

Suzuki DA, Keller EL (1984) Visual signals in the dorsolateral pontine nucleus of the alert monkey: their relationship to smooth-pursuit eye movements. Exp Brain Res 53:473-478.

Suzuki DA, May JG, Keller EL, Yee RD (1990) Visual motion response properties of neurons in dorsolateral pontine nucleus of alert monkey. J Neurophysiol 63:37-59.

Tanaka M, Fukushima K (1998) Neuronal responses related to smooth pursuit eye movements in the periarcuate cortical area of monkeys. J Neurophysiol 80:28-47.

Tanaka M, Lisberger SG (2000) Context-dependent smooth eye movements evoked by stationary visual stimuli in trained monkeys. J Neurophysiol 84:1748-1762.

Tanaka M, Lisberger SG (2001) Regulation of the gain of visually guided smooth-pursuit eye movements by frontal cortex. Nature 409:191-194.

Tanaka M, Lisberger SG (2002a) Enhancement of multiple components of pursuit eye movement by microstimulation in the arcuate frontal pursuit area in monkeys. J Neurophysiol 87:802-818.

Tanaka M, Lisberger SG (2002b) Role of arcuate frontal cortex of monkeys in smooth pursuit eye movements. I. Basic response properties to retinal image motion and position. J Neurophysiol 87:2684-2699.

Tanaka M, Lisberger SG (2002c) Role of arcuate frontal cortex of monkeys in smooth pursuit eye movements. II. Relation to vector averaging pursuit. J Neurophysiol 87:2700-2714.

Tian JR, Lynch JC (1996) Functionally defined smooth and saccadic eye movement subregions in the frontal eye field of Cebus monkeys. J Neurophysiol 76:2740-2753.

Tian JR, Lynch JC (1997) Subcortical input to the smooth and saccadic eye movement subregions of the frontal eye field in Cebus monkey. J Neurosci 17:9233-9247. 